\title{
CANCER
}

\section{Excessive alcohol consumption favours high risk polyp or colorectal cancer occurrence among patients with adenomas: a case control study}

\author{
M Bardou, S Montembault, V Giraud, A Balian, E Borotto, C Houdayer, F Capron, \\ J-C Chaput, S Naveau
}

See end of article for authors' affiliations

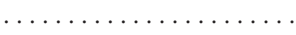

Correspondence to: M Bardou, Service Hépato-gastroentérologie, Hôpital Antoine Béclère, 157, rue de la Porte de Trivaux, F-92141 Clamart Cedex, France; Marc.Bardou@u-bourgogne.fr

Accepted for publication 1 May 2001

\begin{abstract}
Background and aims: Excessive alcohol consumption is a risk factor for developing colorectal adenomas. This study aimed to investigate the influence of excessive alcohol consumption on the occurrence of high risk polyps (adenoma $\geqslant 10 \mathrm{~mm}$, villous component, high grade dysplasia) or colorectal cancer among patients with at least one colonic adenoma.

Patients and methods: Three groups of patients with at least one colorectal adenoma were included in a case control study: 401 heavy drinkers (group HD, mean daily alcohol intake 117 (SD 4) g/day for a mean duration of 22 (SD 0.6) years), aged $57(0.5)$ years $(78 \%$ men); 152 patients suffering from irritable bowel syndrome (IBS), aged 61 (0.9) years (57\% male); and 108 patients with a family history $(\mathrm{FH})$ of colorectal adenoma or cancer, aged $55(1)$ years $(64 \%$ male). Exclusion criteria were: anaemia, haematochezia, personal history of colorectal adenoma or cancer, and for groups HD and IBS a family history of colorectal adenoma and/or cancer. Relative risks were estimated by the odds ratio (OR) using a logistic regression model and were expressed with $95 \%$ confidence interval (CI). Results: After age and sex adjustment, the likelihood of having an adenoma $\geqslant 10 \mathrm{~mm}$ was higher in group HD than in the IBS group (OR 1.8, 95\% Cl (1.2-2.7)) and the likelihood of having high risk adenomas or cancer was higher in group HD compared with the IBS group (OR 1.6, $95 \% \mathrm{Cl}$ $(1.2-2.1))$ and the $\mathrm{FH}$ group although this was not significant $(\mathrm{OR} 1.6,95 \% \mathrm{Cl}(0.97-2.6)(\mathrm{p}=0.081)$; $90 \% \mathrm{Cl}(1.03-2.4))$. After age and sex adjustment, the likelihood of having an adenoma with high grade dysplasia or cancer was higher in group HD than in the IBS group (OR 1.7,95\% CI (1.02-2.8)) or group $\mathrm{FH}$, although this was not significant (OR 3.7, 95\% Cl (0.98-15) (p=0.076); $90 \% \mathrm{Cl}(1.10-$ 12.47)1.

Conclusion: In patients with at least one colorectal adenoma, excessive alcohol consumption increases the likelihood of developing high risk adenomas or colorectal cancer.
\end{abstract}

con he results of previous studies on the association of alcohol consumption and the risk of developing colorectal carcinoma were controversial. Some reported a positive association, ${ }^{12}$ particularly with beer consumption, ${ }^{3-6}$ whereas others did not. ${ }^{7-9}$

A recent meta analysis ${ }^{10}$ of alcoholic beverage consumption in relation to risk of colorectal cancer supported the presence of a weak association. The association did not vary according to sex or site within the large bowel, and the evidence supporting beverage specificity was not conclusive.

The relation between alcohol consumption and risk of developing colorectal adenomas has been demonstrated previously by several authors. Cope et al found that the risk of polyps was increased three times for drinkers who did not smoke and 12 times for both drinkers and smokers compared with the risk of total abstainers. ${ }^{11}$ Naveau et al found that alcoholism and cirrhosis were two independent risk factors for colorectal adenomatous polyps after successive adjustments for sex, age, smoking, and serum cholesterol levels. ${ }^{12}$

In experimental models of rectal carcinogenesis, the results were also conflicting as some authors found that high chronic intake of alcohol significantly increased the number of rectal tumours ${ }^{13}$ and selectively stimulated epithelial cell proliferation in the rectum ${ }^{14}$ whereas other authors found no effect on tumorigenesis or even suppressed tumour growth. ${ }^{15} 16$

The colorectal adenoma-carcinoma sequence is now widely accepted and, according to the hypothesis of Hill and colleagues, ${ }^{17}$ factors causing the development of adenomas per se differ from those that influence the growth of small adeno- mas and from those that induce malignant changes. Some studies suggested that tobacco might be associated with adenomas, and alcohol with the risk of large adenomas ${ }^{18}{ }^{19}$ and colorectal cancer, ${ }^{20}$ even if recent studies suggested that neither alcohol nor tobacco play a major role in the formation or growth of adenomas. ${ }^{9}$ It has been suggested that alcohol does not have a direct carcinogenic effect but may be involved in the early stage of tumorigenesis by promoting growth of the adenoma. $^{21}$

We have undertaken a case control study to investigate the hypothesis that alcoholism may increase the risk of having a high risk ( $\geqslant 10 \mathrm{~mm}$ in size, high grade dysplasia, villous component) colorectal adenoma or a colorectal cancer.

\section{MATERIAL AND METHODS}

Patients

All patients older than 40 years who were referred to our unit between January 1987 and December 1996 for excessive alcohol consumption (heavy drinkers (group HD) $>50 \mathrm{~g}$ of alcohol/day for the year before admission) underwent screening colonoscopy if they had no contraindications. Two other groups of patients who drank less than $30 \mathrm{~g}$ /day were included during the same period: patients who were undergoing colonoscopy because of a family history (group FH) of colorectal

Abbreviations: IBS, irritable bowel syndrome; OR, odds ratio 
Table 1 Age and sex characteristics of patients with at least one colorectal adenoma in the irritable bowel syndrome (IBS), family history (FH), and heavy drinkers (HD) groups

\begin{tabular}{lllll}
\hline & IBS group & FH group & HD group & p Value* \\
\hline $\mathrm{n}$ & 152 & 108 & 401 & \\
Age (y) (mean (SD)) & $61(0.9)$ & $55(1)$ & $57(0.5)$ & $<0.05$ \\
Male/Female ratio & $87 / 65(57 \%)$ & $69 / 39(64 \%)$ & $313 / 88(78 \%)$ & $<0.001$ \\
\hline
\end{tabular}

${ }^{*} \chi^{2}$ test or $\mathrm{F}$ test from ANOVA.

cancer or adenomatous polyps (no patient had a family history of adenomatous polyposis coli) and patients who were suffering from irritable bowel syndrome (IBS). IBS was diagnosed using the Rome II criteria. ${ }^{22}$

Information on alcohol consumption was recorded using a specific standardised 25 item questionnaire, which has recently been revalidated..$^{23}$ Patients were asked about their drinking habits a few days before colonoscopy during consultation for outpatients or during hospitalisation for inpatients. The questionnaires were completed in the same standardised way by a senior gastroenterologist in our unit. We did not use a self administrated questionnaire as French people are not familiar with them. Nalpas and colleagues ${ }^{24}$ described recently in a French population that anonymous self administrated questionnaires were associated with a high level of incomplete questionnaires or with responses that were unusable. This 25 item questionnaire was used in the same way for outpatients and inpatients in the three groups. The same senior gastroenterologists interviewed outpatients and inpatients. Patients were asked about their drinking habits over the previous five years before admission to hospital for alcoholic disorders and total duration of alcohol abuse. The average daily intake was calculated for each beverage (beer, wine, aperitifs) and expressed in grams of pure ethanol. Total daily consumption of ethanol was obtained by adding the amounts consumed for each type of beverage. It took approximately 15 minutes to complete a questionnaire. The patient's family was also interviewed if possible.

All patients in the three groups were asked about their personal and family history of colorectal adenomas or cancer, and about their bowel habits, based on the Rome criteria, to avoid overlapping of the three groups. Criteria for exclusion were anaemia, haematochezia, familial polyposis, inflammatory bowel disease, personal history of colorectal adenomas or cancer and, except for the FH group, family history of colorectal adenomas or cancer. Alcoholic patients were not included if they had uncompensated cirrhosis. Patients were included in the study only if colonoscopy reached the caecum.

During the 10 year period of the study, among the 4517 patients who were referred to our unit for a screening colonoscopy without any clinical or biological symptoms of intestinal disease, 661 patients were entered into the study because of the pressence of at least one colorectal adenoma. A total of 401 patients were included in the HD group (mean daily alcohol intake 117 (SD 4) g/day for a mean duration of 22 (SD 0.6 ) years), 152 in the IBS group, and 108 in the FH group. Age and sex distributions were different between the three groups (table 1).

\section{Cases and controls}

As the exposing factor being studied was excessive alcohol consumption, three case control studies were performed.

- In the first study, cases $(\mathrm{n}=84)$ were patients with at least one adenomatous polyp larger than $10 \mathrm{~mm}$, and controls $(\mathrm{n}=489)$ were those with adenomatous polyps $<10 \mathrm{~mm}$ and without villous contingent high grade dysplasia or cancer.
- In the second study, cases $(n=172)$ were patients with at least one high risk colorectal polyp ( $\geqslant 10 \mathrm{~mm}$ and/or with a villous component and/or with high grade dysplasia) or a histologically proved colorectal cancer, and controls $(\mathrm{n}=489)$ were those with adenomas $<10 \mathrm{~mm}$ and without high grade dysplasia, a villous component, or colorectal cancer.

- In the third study, cases $(n=37)$ were patients with high grade dysplasia or colorectal cancer, and controls $(n=624)$ were those with adenomatous polyps, irrespective of size, and no high grade dysplasia or colorectal cancer.

All colonoscopies had to reach at least the caecum and were performed by a senior endoscopist. In all patients with at least one adenoma of the colon, the location and number of adenomas was recorded as was the location of high risk adenomas or cancer.

Histopathological diagnosis was performed in the anathomopathology unit of our hospital and the degree of dysplasia was classified according to the World Health Organisation classification system. ${ }^{25}$

\section{Statistical analysis}

Univariate analysis studies were performed using the $\chi^{2}$ method for dichotomous variables and the Student's $t$ test for continuous variables.

Relative frequencies in the three groups of patients (IBS, FH and HD) of cases and controls were compared using the global $\chi^{2}$ method.

Relative risks of adenomas $\geqslant 10 \mathrm{~mm}$, high risk adenoma or cancer, and high grade dysplasia or cancer, according to study group, were estimated by odds ratios (ORs) adjusted for age and sex using a stepwise logistic regression. In this model, if the value of the probability level is less than some predefined alpha level, say 0.1 , then the variable is said to be statistically significant. The statistical significance of each studied variable was tested using the maximum likelihood method.

\section{RESULTS}

\section{Characteristics of the adenomas}

Adenomas $\geqslant 10 \mathrm{~mm}$ were found in 122 patients: 84 of these 122 patients had adenomas $\geqslant 10 \mathrm{~mm}$ without villous contingent or high grade dysplasia, nine patients in the IBS group, 16 in the FH group, and 59 in the HD group. Adenomas with a villous component were found in 57 patients $(13,5$, and 39 patients in the IBS, FH, and HD groups, respectively). Adenomas with high grade dysplasia were found in 28 patients (three, two, and 23 patients in the IBS, FH, and HD groups, respectively). High risk adenoma ( $\geqslant 10 \mathrm{~mm}$ or with a villous contingent or with severe dysplasia) were found in 172 patients $(25,23$, and 124 patients in the IBS, FH, and HD groups, respectively). Colorectal cancer was found in 12 patients (three, none, and nine patients in the IBS, FH, and HD groups, respectively). Most (68.64\%) of the high risk colorectal adenomas were located in the distal colon (left colon, sigmoid, and rectum). A total of $90 \%$ of patients had a single high risk colorectal adenoma while $3 \%$ had three or more high risk adenomas. 
Table 2 Group distribution between cases and controls for adenomas $>10 \mathrm{~mm}$

\begin{tabular}{|c|c|c|c|}
\hline & $\begin{array}{l}\text { Cases } \\
\text { Adenomas } \\
>10 \mathrm{~mm} \\
(\mathrm{n}=84)\end{array}$ & $\begin{array}{l}\text { Controls } \\
\text { Adenomas } \\
<10 \mathrm{~mm} \\
(\mathrm{n}=489)\end{array}$ & $\mathrm{p}$ Value \\
\hline Age (y) (mean (SD)) & $59(1)$ & $57(0.5)$ & $<0.05$ \\
\hline $\begin{array}{l}\text { Male/female ratio } \\
\text { Patient groups }\end{array}$ & $61 / 23(73 \%)$ & $343 / 146(70 \%)$ & NS \\
\hline IBS & $9(11 \%)$ & $127(26 \%)$ & \\
\hline $\mathrm{FH}$ & $16(19 \%)$ & $85(17 \%)$ & $<0.01$ \\
\hline$H D$ & $59(70 \%)$ & $277(57 \%)$ & \\
\hline
\end{tabular}

The $p$ value is given for the comparison by global $\chi^{2}$ method of the relative frequency in the three groups of patients (irritable bowel syndrome (IBS), family history (FH), and heavy drinkers (HD) groups) of cases and controls. The number of patients (cases plus controls $=571$ ) is less than the total number of patients (601) because cases with adenomas $>10 \mathrm{~mm}$ and high grade dysplasia or a villous contingent were excluded from this part of the study.

Table 3 Group distribution between cases and controls for high risk adenomas or colorectal cancer

\begin{tabular}{lccl}
\hline & $\begin{array}{l}\text { Cases } \\
\text { High risk } \\
\text { adenomas } \\
\text { or cancer } \\
(\mathrm{n}=172)\end{array}$ & $\begin{array}{l}\text { Controls } \\
\text { No high risk } \\
\text { adenomas } \\
\text { or cancer } \\
(\mathrm{n}=489)\end{array}$ & p Value \\
\hline $\begin{array}{l}\text { Age (mean (SD)) } \\
\text { Male/female ratio }\end{array}$ & $\begin{array}{c}59(0.9) \\
126 / 46(73 \%)\end{array}$ & $\begin{array}{c}57(0.5) \\
343 / 146(70 \%)\end{array}$ & $\begin{array}{l}0.049 \\
\text { NS }\end{array}$ \\
$\begin{array}{l}\text { Patient groups } \\
\text { IBS }\end{array}$ & $25(15 \%)$ & $127(26 \%)$ & \\
FH & $23(13 \%)$ & $85(17 \%)$ & 0.00096 \\
HD & $124(72 \%)$ & $277(57 \%)$ & \\
\hline
\end{tabular}

The $p$ value is given for the comparison by global $\chi^{2}$ method of the relative frequency in the three groups of patients (irritable bowel syndrome (IBS), family history (FH), and heavy drinkers (HD) groups) of cases (patients with at least one high risk adenoma or a cancer) and controls (patients with a least one colorectal adenoma but without high risk adenomas or cancer).

\section{Results of analysis}

In the first case control study, we examined the relative frequency in the three risk groups (IBS, FH, HD) of patients with adenomas $\geqslant 10 \mathrm{~mm}$ but without high grade dysplasia or a villous contingent (cases) and patients without adenomas $\geqslant 10 \mathrm{~mm}$ and without high grade dysplasia or a villous contingent (controls). This relative frequency was significantly different $(\mathrm{p}<0.01)$ between cases $(\mathrm{n}=84)$ and patients with adenomas $<10 \mathrm{~mm}$ (controls, $\mathrm{n}=489$ ). Heavy drinking was more frequent $(70 \%)$ in cases than in controls $(57 \%)$ (table 2).

After age and sex adjustment, the likelihood of having an adenoma $\geqslant 10 \mathrm{~mm}$ was higher in group HD than in group IBS (OR 1.8 (95\% confidence interval (CI) 1.2-2.7); p<0.05). After age and sex adjustment, the likelihood of having an adenoma $\geqslant 10 \mathrm{~mm}$ was not significantly different between groups HD and FH (OR 1.14 (95\% CI 0.7-2.0); NS).

In the second case control study, we examined the relative frequency in the three risk groups of cases with a high risk adenoma (adenomas $\geqslant 10 \mathrm{~mm}$ or with high grade dysplasia or a villous component) or colorectal cancer, and of controls. As in the first part of our study, the relative frequency in the three groups of patients was significantly $(p<0.001)$ different between patients with high risk adenomas or colorectal cancer (cases, $\mathrm{n}=172$ ) and those without high risk adenomas or cancer (controls, $\mathrm{n}=489$ ). Heavy drinkers were more frequent $(72 \%)$ in cases than in controls $(57 \%)$ (table 3$)$.

After age and sex adjustment, the likelihood of having a high risk adenoma or colorectal cancer was higher in group HD than in group IBS (OR 1.6 (95\% CI 1.2-2.1); $<<0.001$ ) and
Table 4 Group distribution between cases and controls for adenomas with high grade dysplasia or colorectal cancer

\begin{tabular}{llll}
\hline & $\begin{array}{l}\text { Cases } \\
\text { High grade } \\
\text { dysplasia } \\
\text { or cancer } \\
(\mathrm{n}=37)\end{array}$ & $\begin{array}{l}\text { Controls } \\
\text { No high grade } \\
\text { dysplasia } \\
\text { or cancer } \\
(\mathrm{n}=624)\end{array}$ & $\mathrm{p}$ Value \\
\hline $\begin{array}{l}\text { Age (mean (SD)) } \\
\begin{array}{l}\text { Male/female ratio } \\
\text { Patient groups }\end{array}\end{array}$ & $61(2)$ & $57(0.5)$ & 0.046 \\
IBS & $50 / 7(81 \%)$ & $439 / 185(70 \%)$ & $\mathrm{NS}$ \\
FH & $2(14 \%)$ & $147(24 \%)$ & \\
HD & $30(81 \%)$ & $371(59 \%)$ & 0.048 \\
\hline
\end{tabular}

The $p$ value is given for the comparison by global $\chi^{2}$ method of the relative frequency in the three groups of patients (irritable bowel syndrome (IBS), family history (FH), and heavy drinkers (HD) groups) of cases (patients with at least one adenoma with high grade dysplasia or a cancer) and controls (patients with a least one colorectal adenoma but without high grade dysplasia or cancer)

was also higher, although not significantly at the 5\% level, in group HD than in group FH (OR 1.6 (95\% CI 0.97-2.6) $(\mathrm{p}=$ $0.081), 90 \%$ CI (1.02-2.3)).

In the third of our case control studies, we examined the relative frequency in the three risk groups of patients with adenomas with high grade dysplasia or colorectal cancer (cases, $\mathrm{n}=37$ ) and patients without adenomas with high grade dysplasia or colorectal cancer (controls, $n=624$ ). The relative frequency in the three groups was statistically different between cases and controls $(p<0.05)$. Heavy drinking was more frequent $(81 \%)$ in cases with high grade dysplasia adenomas or colorectal cancer than in controls (59\%) (table 4).

After age and sex adjustment, the likelihood of having a high grade dysplasia adenoma or a colorectal cancer was higher in group HD than in group IBS (OR 1.7 (95\% CI 1.02$2.8) ; \mathrm{p}<0.05$ ) and, although without being statistically significant at the 5\% level, than in group FH (OR 3.7 (95\% CI $0.98-15)(\mathrm{p}=0.076), 90 \%$ CI $(1.10-12.47))$.

\section{Influence of cirrhosis}

In the HD group, we evaluated the influence of cirrhosis in the three case control studies and found that cirrhotic patients were not significantly more numerous between cases and controls in any of the three studies performed (table 5).

\section{DISCUSSION}

We have shown a relationship between alcoholism and the risk of high risk adenomas or colorectal cancer. To our knowledge, this is the first study that has investigated high risk adenomas or cancer among alcoholic patients with endoscopically proved adenomas compared with two other populations, one with a risk higher than in the general population (patients with a family history of colorectal tumours) and one with a risk thought to be at least equal to the general population (those suffering from IBS). ${ }^{26}$ The association between colorectal cancer and alcohol consumption is controversial. A meta analysis of the relation between alcohol and colorectal cancer found only a small but significant effect. ${ }^{10}$

A recent case control study in Argentina found an OR for colorectal cancer of 2.8 (1.6-5.1) for drinkers in comparison with alcohol abstainers, with a significant trend in risk with dose. ${ }^{27}$ The association was observed for wine, as well as for beer and spirits.

The association between alcohol consumption and large adenomas has already been proved by Boutron and colleagues. ${ }^{18}$ They concluded that alcohol may be involved in the second step of the adenoma-carcinoma sequence by 
Table 5 Relation between cirrhosis and: adenomas $>10 \mathrm{~mm}$; high risk adenomas or cancer; and high grade dysplasia or cancer among alcoholic patients

\begin{tabular}{llll}
\hline Cirrhosis & Adenomas $>10 \mathrm{~mm}(\mathrm{n}=59)$ & Adenomas $<10 \mathrm{~mm}(\mathrm{n}=276)$ & p Value \\
& $20(34 \%)$ & $105(38 \%)$ & NS \\
Cirrhosis & High risk adenoma or cancer $(\mathrm{n}=124)$ & No high risk adenoma or cancer $(\mathrm{n}=277)$ & NS \\
& $51(41 \%)$ & $106(38 \%)$ & \\
Cirrhosis & High grade dysplasia or cancer $(\mathrm{n}=30)$ & No high grade dysplasia or cancer $(\mathrm{n}=371)$ & NS \\
\hline
\end{tabular}

Per cent values represent the number of patients with proven cirrhosis (cirrhosis was assessed either on a liver biopsy or using clinical and biological signs) among the total number of patients in each subgroup of the heavy drinkers.

promotion of growth of the adenoma, and that alcohol acts as an early promoter of colorectal carcinogenesis but plays no role in malignant changes.

The mechanism by which alcohol might promote colorectal cancer is still controversial. Alcohol in itself would seem to have no direct carcinogenic effect. Its metabolite acetaldehyde has been proved to be cytotoxic. ${ }^{28}$

A direct action of acetaldehyde on the faecal microflora has been suggested..$^{29}{ }^{30}$ Seitz et al have demonstrated in an animal model that the intestinal flora may be involved in acetaldehyde production as germ free rats had significantly lower acetaldehyde concentrations in the rectum (84 (11) v 234 (33) $\mathrm{nmol} / \mathrm{g} / \mathrm{colon} ; \mathrm{p}<0.01$ ) and in the caecum (59 (13) $v 121$ (33) $\mathrm{nmol} / \mathrm{g} /$ colon; $\mathrm{p}<0.05$ ) compared with conventional animals, and that alcohol feeding induced rectal but not caecal hyperregeneration. ${ }^{30}$ Acetaldehyde can react with various intracellular and extracellular proteins to form both stable and unstable condensation products. Several proteins involved in these reactions have been shown to form cross links with acetaldehyde, including albumin, ${ }^{31}$ tubulin, ${ }^{32}$ haemoglobin, ${ }^{33}$ collagen, ${ }^{34}$ and cytochrome P-450IIE $1 .{ }^{35}$ Acetaldehyde-protein adduct formation may lead to cellular damage $e^{36}$ or dysfunction through alteration of biological protein function or adduct triggered immunological effects. ${ }^{37}$ Holstege et al have hypothesised that only on their extracellular location, acetaldehyde modified epitopes could serve as neoantigens and thereby elicit humoral or cellular immune responses. ${ }^{38}$

Ki-ras mutations are thought to be early events in the carcinogenic process leading to colon tumours. It has been reported that alcohol may be involved in the disease pathway of colon tumours represented by specific Ki-ras mutations. ${ }^{39}$

Ethanol may increase the risk of adenomatous polyps by interfering with dietary habits, and the relation between ethanol and adenomatous polyps could be the result of confounding by unspecified dietary factors such as folate or vitamin $\mathrm{C}$ as alcoholic patients often have a low fruit and vegetable diet. Folate, which is plentiful in vegetables and fruits, may be protective against colorectal cancer. A recent nested case control study by Kato and colleagues ${ }^{40}$ found that the risk of colorectal cancer in patients within the highest quartile for serum folate was half that of those in the lowest quartile. They also found that the risk of colorectal cancer was almost twice as high in subjects with below median serum folate and above median total alcohol intake compared with those with above median serum folate and below median alcohol consumption. This may suggest that alcohol intake and folate deficiency have a synergistic action in the promotion of colorectal carcinoma, particularly in alcoholic patients who usually have a low folate and vitamin $\mathrm{C}$ intake and a high fat intake. ${ }^{41}$ This has recently been confirmed in a large epidemiological study. ${ }^{42}$ Multivitamin use and folate intake have been reported to have a protective effect in colon cancer in women only after long use. ${ }^{43}$ We might hypothesise that the risk of colorectal high risk adenomas would be increased only after many years of a low folate diet. Our alcoholic patients were drinking more than $50 \mathrm{~g} /$ day for a mean duration of $22(0.6)$ years.
We did not specifically study the role of tobacco but this is another risk factor in these results, as we previously found ${ }^{12}$ that alcoholic patients are more likely to be current smokers than patients suffering from IBS. This is in agreement with Faivre and colleagues ${ }^{19}$ who found that the proportion of large adenomas in men which could be attributed to combined alcohol and tobacco consumption was $78 \%$.

We did not study the role of cholesterol but it needs to be discussed as we have previously observed that serum cholesterol concentration was lower in patients with adenomas than in patients without adenomas. ${ }^{12}$ In the same study, we showed that alcoholism was independently associated with the risk of having colorectal adenomas.

The ORs after adjustment for age and sex were in agreement with the findings that alcoholism may be involved in the severity of colonic adenomas as the relative risk of heavey drinkers having a large adenoma, a high risk adenoma or cancer, or a high grade dysplasia or cancer was significantly increased compared with IBS patients (ORs 1.8, 1.6, and 1.7, respectively). Comparison between the $\mathrm{HD}$ and $\mathrm{FH}$ groups showed that there was a trend towards an increased risk for high risk adenomas or cancer and for high grade dysplasia or cancer in group HD compared with group FH, but this was not statistically significant at the $5 \%$ level.

In conclusion, in our study alcoholism was a risk factor for the development of high risk adenomas or colorectal cancer. This suggest that alcoholic patients may benefit from a screening policy of colonic adenomatous polyps when their liver function allows.

Authors' affiliations

M Bardou, S Montembault, V Giraud, A Balian, E Borotto, C Houdayer, J-C Chaput, S Naveau, Hepatogastroenterology Unit Antoine Béclère Hospital, 157, rue de la Porte de Trivaux F-92 141 Clamart Cedex, France

F Capron, Anatomopathology Unit, Antoine Béclère Hospital, 157, rue de la Porte de Trivaux F-92141 Clamart Cedex, France

\section{REFERENCES}

1 Pollack ES, Nomura AM, Heilbrun LK, et al. Prospective study of alcoho consumption and cancer. N Engl J Med 1984;310:617-21.

2 Wu AH, Paganini-Hill A, Ross RK, et al. Alcohol, physical activity and other risk factors for colorectal cancer: a prospective study. $\mathrm{Br} J$ Cancer 1987; 55:687-94.

3 Enstrom JE. Colorectal cancer and beer drinking. Br J Cancer 1977;35:674-83.

4 Kune S, Kune GA, Watson LF. Case-control study of alcoholic beverages as etiological factors: the Melbourne Colorectal Cancer Study. Nutr Cancer 1987:9:43-56.

5 Sandler RS, Lyles CM, McAuliffe C, et al. Cigarette smoking, alcohol, and the risk of colorectal adenomas. Gastroenterology 1993; 104: 1445-51

6 Kikendall JW, Bowen PE, Burgess MB, et al. Cigarettes and alcohol as independent risk factors for colonic adenomas. Gastroenterology 1989:97:660-4.

7 Bielke E. Epidemiologic studies of cancer of the stomach, colon, and rectum, with special emphasis on the role of diet. Scand J Gastroenterol Suppl 1974;31:1-235.

8 Nagata C, Shimizu H, Kametani M, et al. Cigarette smoking, alcohol use, and colorectal adenoma in Japanese men and women (in process citation). Dis Colon Rectum 1999;42:337-42. 
9 Breuer-Katschinski B, Nemes K, Marr A, et al. Alcohol and cigarette smoking and the risk of colorectal adenomas. Dig Dis $\mathrm{Sci}$ 2000;45:487-93.

10 Longnecker MP, Orza M, Adams ME, et al. A meta-analysis of alcoholic beverage consumption in relation to risk of colorectal cancer. Cancer Causes Control 1990;1:59-68.

11 Cope GF, Wyatt JI, Pinder IF, et al. Alcohol consumption in patients with colorectal adenomatous polyps. Gut 1991;32:70-2.

12 Naveau S, Chaput JC, Bedossa P, et al. Cirrhosis as an independent risk factor for colonic adenomas. Gut 1992;33:535-40.

13 Seitz HK, Czygan P, Simanowski U, et al. Stimulation of chemically induced rectal carcinogenesis by chronic ethanol ingestion. Alcohol Alcohol 1985;20:427-33

14 Simanowski UA, Seitz HK, Baier B, et al. Chronic ethanol consumption selectively stimulates rectal cell proliferation in the rat. Gut 1986;27:278-82

15 Nelson RL, Samelson SL. Neither dietary ethanol nor beer augments experimental colon carcinogenesis in rats. Dis Colon Rectum 1985;28:460-2.

16 Hamilton SR, Sohn OS, Fiala ES. Effects of timing and quantity of chronic dietary ethanol consumption on azoxymethane-induced colonic carcinogenesis and azoxymethane metabolism in Fischer 344 rats. Cancer Res 1987;47:4305-11.

17 Hill MJ, Morson BC, Bussey HJ. Aetiology of adenoma-carcinoma sequence in large bowel. Lancet 1978;1:245-7.

18 Boutron MC, Faivre J, Dop MC, et al. Tobacco, alcohol, and colorectal tumors: a multistep process. Am J Epidemiol 1995;141:1038-46.

19 Faivre J, Boutron MC, Senesse P, et al. Environmental and familial risk factors in relation to the colorectal adenoma-carcinoma sequence: results of a case-control study in Burgundy (France). Eur J Cancer Prev 1997:6:127-31

20 Yamada K, Araki S, Tamura M, et al. Case-control study of colorecta carcinoma in situ and cancer in relation to cigarette smoking and alcohol use (Japan). Cancer Causes Control 1997;8:780-5.

21 Boutron-Ruault MC. Alimentation et cancerogénèse colorectale: éléments pour une prévention primaire. Gastroenterol Clin Biol 1998;22:S12-20.

22 Drossman DA, Thompson GW, Talley NJ, et al. Identification of subgroups of functional gastrointestinal disorders. Gastroenterol Int 1990;3:159-72.

23 Naveau S, Chemla P, Chaput J-C. Validité relative d'un questionnaire évaluant la consommation d'alcool d'un groupe de buveurs hospitalisés. Cah Nutr Diet 2000;35:121-5.

24 Nalpas B, Zylberberg H, Dubois F, et al. (Prevalence of infection by hepatitis viruses in a rural area. Analysis according to risk factors and alcohol consumption). Gastroenterol Clin Biol 2000;24:536-40.

25 Morson B. Polyps and cancer of the large bowel. West J Med 1976;125:93-9

26 Garcia Rodriguez LA, Ruigomez A, Wallander MA, et al. Detection of colorectal tumor and inflammatory bowel disease during follow-up of patients with initial diagnosis of irritable bowel syndrome. Scand J Gastroenterol 2000:35:306-11.

27 Munoz SE, Navarro A, Lantieri M, et al. Alcohol, methylxanthine-containing beverages, and colorectal cancer in Cordoba, Argentina. Eur J Cancer Prev 1998;7:207-13.

28 Lauterburg BH, Bilzer M. Mechanisms of acetaldehyde hepatotoxicity. J Hepatol 1988;7:384-90.

29 Levitt MD, Doizaki W, Levine AS. Hypothesis: metabolic activity of the colonic bacteria influences organ injury from ethanol. Hepatology 1982;2:598-600.

30 Seitz HK, Simanowski UA, Garzon FT, et al. Possible role of acetaldehyde in ethanol-related rectal cocarcinogenesis in the rat. Gastroenterology 1990;98:406-13.

31 Lumeng L, Durant PJ. Regulation of the formation of stable adducts between acetaldehyde and blood proteins. Alcohol 1985:2:397-400.

32 Tuma DJ, Jennett RB, Sorrell MF. The interaction of acetaldehyde with tubulin. Ann N Y Acad Sci 1987;492:277-86.

33 Stevens VJ, Fantl WJ, Newman CB, et al. Acetaldehyde adducts with hemoglobin. J Clin Invest 1981;67:361-9.

34 Behrens UJ, Ma XL, Bychenok S, et al. Acetaldehyde-collagen adducts in $\mathrm{CCl} 4$-induced liver injury in rats. Biochem Biophys Res Commun 1990;173:111-19.

35 Behrens UJ, Hoerner M, Lasker JM, et al. Formation of acetaldehyde adducts with ethanol-inducible P450IIE 1 in vivo. Biochem Biophys Res Commun 1988;154:584-90.

36 Mauch TJ, Donohue TM Jr, Zetterman RK, et al. Covalent binding of acetaldehyde selectively inhibits the catalytic activity of lysine-dependent enzymes. Hepatology 1986;6:263-9.

37 Hoerner M, Behrens UJ, Worner TM, et al. The role of alcoholism and liver disease in the appearance of serum antibodies against acetaldehyde adducts. Hepatology 1988;8:569-74.

38 Holstege A, Bedossa P, Poynard T, et al. Acetaldehyde-modified epitopes in liver biopsy specimens of alcoholic and nonalcoholic patients: localization and association with progression of liver fibrosis. Hepatology 1994; 19:367-74.

39 Slattery ML, Curtin K, Anderson K, et al. Associations between dietary intake and Ki-ras mutations in colon tumors: a population-based study. Cancer Res 2000;60:6935-41.

40 Kato I, Dnistrian AM, Schwartz $M$, et al. Serum folate, homocysteine and colorectal cancer risk in women: a nested case-control study. $\mathrm{Br} J$ Cancer 1999;79:1917-22

41 Hoff G, Moen IE, Trygg K, et al. Epidemiology of polyps in the rectum and sigmoid colon. Evaluation of nutritional factors. Scand J Gastroenterol 1986:21:199-204.

42 Su LJ, Arab L. Nutritional status of folate and colon cancer risk: evidence from NHANES I epidemiologic follow-up study. Ann Epidemiol $2001 ; 11: 65-72$.

43 Giovannucci E, Stampfer M, Colditz GA, et al. Multivitamin use, folate, and colon cancer in women in the Nurses' Health Study. Ann Intern Med 1998; 129:517-24. 